\title{
Results of Novel Strategies for Treatment of Wilms' Tumor
}

\author{
Silvio Tucci Jr, Adauto J. Cologna, Haylton J. Suaid, Elvis T. Valera, Luis F. Tirapelli, Edson L. \\ Paschoalin, Antonio C. Martins
}

Division of Urology, Ribeirao Preto Medical School, University of Sao Paulo, Ribeirao Preto, Sao Paulo, Brazil

\begin{abstract}
Objective: To evaluate treatment outcomes in Wilms' tumor (WT).

Materials and Methods: We studied 53 children with median age of 2 years with WT, stages I-19, II-14, III-12, IV-6 and V2. Treatment consisted of surgical excision plus adjuvant (40 children) or neoadjuvant and adjuvant chemotherapy (unresectable tumor, $\mathrm{n}=8$, or caval tumor extension, $\mathrm{n}=5$ ). Chemotherapy and radiotherapy followed protocols of Brazilian Wilms' Tumor Study Group excepting 16 cases with stage I disease that received a short duration postoperative treatment with vincristine (VCR - 11 doses) and dactinomycin (AMD - 4 doses). Relapsed WT was treated with multiagent regimens including cisplatin/carboplatin, cyclophosphamide, ifosfamide and etoposide. One patient with resistant relapsed WT was treated by high-dose conditioning chemotherapy with stem cell rescue.

Results: Overall and disease-free survival rates at 5 years were respectively $88.2 \pm 5.0 \%$ and $76.7 \pm 6.6 \%$. Short duration therapy for stage I tumor showed a disease-free survival rate of $100 \%$ in a median time of 101 months (range 14 to 248 months). Overall and disease-free survival of 10 patients with recurrent WT at 5 years was $42.8 \%$. The child treated with high-dose chemotherapy plus stem cell transplant is alive without evidence of disease 84 months from relapse.

Conclusion: The postoperative chemotherapy in stage I disease can be reduced without compromising the cure rate. The treatment of unfavorable stage III and IV disease or relapsed tumor remains a challenge.
\end{abstract}

Key words: Wilms' tumor; surgery; chemotherapy; relapse

Int Braz J Urol. 2007; 33: 195-203

\section{INTRODUCTION}

The multidisciplinary management of Wilms' tumor (WT) led to a striking improvement of patient outcome in the last decades. Now, an increasing consideration is given to determine the minimal therapy needed to cure low-risk tumors $(1,2)$. Recent WT trials of the International Society of Pediatric Oncology (SIOP) and United Kingdom Children's Cancer Study
Group (UKCCSG) has shown that chemotherapy with vincristine (VCR) or a combination of VCR and dactinomycin (AMD) for stage I favorable histology tumor can be reduced without compromising survival rates, but there is no agreement on the matter (1-4). At the same time, researchers are looking for novel strategies such as treatment intensification for children with high-risk tumors (5). The use of modern intensive regimens including doxorubicin (ADR), 
cyclophosphamide (CTX), ifosfamide (IFO), carboplatin (CP) and etoposide (ETP) were reported to improve survival rates for patients with favorable relapsed WT from less than $30 \%$ to $50-55 \%$ (5-7). However, children with unfavorable relapsed WT have a high risk of treatment failure $(1,7)$. Few reports on high-dose chemotherapy with autologous stem-cell rescue show variable disease-free survival rates (33 to $60 \%$ ) and it is not clear whether this approach offers any advantages over conventional second line therapies $(1,5,8-10)$.

The aim of this study is to analyze the outcomes in children with WT treated in a single center with special attention to stage I disease and to patients with high-risk recurrent disease.

\section{MATERIALS AND METHODS}

We reviewed the records of 53 children (28 males and 25 females) with WT who were treated at our institution between January 1980 and December 2004. Median patient age was 2 years (range $<1$ to 14). Inclusion criteria were adequate clinical and pathological data, and a follow-up of 1 year or more, except for those who died of the disease. Two patients were excluded from the study because 1 died just after arrival, and another one that died in the 1st postoperative day of a nephrectomy.

Tumor stage and histological subtype (Table1) were defined according to the National Wilms' Tumor Study Group (NWTSG) $(1,11)$.

Surgical treatment for unilateral disease consisted of transperitoneal radical nephrectomy. One patient with stage $\mathrm{V}$ tumor was submitted to bilateral biopsies followed by chemotherapy and then to total unilateral nephrectomy and partial contralateral nephrectomy. Other child with stage $\mathrm{V}$ in whom a very small nodule in the left kidney was not noticed at presentation was treated initially by unilateral right nephrectomy followed by chemotherapy and afterward by partial left nephrectomy. Regional lymph node sampling was obtained in all patients and an associated adrenalectomy was performed in 16 cases, enterectomy in 2 and caval thrombectomy in 5. Forty patients were treated with adjuvant chemotherapy only. Thirteen children with tumors initially deemed unresectable or with intracaval extension received neoadjuvant and adjuvant therapy as follows: stages I - 3, II - 1, III - 4, IV - 3 and V - 1.

With exception of patients with stage I tumor, the routine adjuvant chemotherapy and radiotherapy followed the recommendations of the Brazilian Wilms' Tumor Study Group (12). Most patients with stage I tumor (16/19) received a shorter postoperative treatment with 11 doses of VCR $\left(1.5 \mathrm{mg} / \mathrm{m}^{2}\right.$, weeks 2-11 and 16) and 4 doses of AMD (15-60 $\mu \mathrm{g} / \mathrm{kg}$, weeks $1,6,11$ and 16).

Relapsed WT was treated with multiagent salvage regimens including CIS, CP, CTX, IFO and ETP $(6,7)$. In addition, abdomen radiotherapy was used in 6 cases (10.5 to $30 \mathrm{~Gy}$ ) and lung radiotherapy in 3 (12 to 15 Gy). One patient with WT (metastasis in liver and lungs) resistant to salvage regimen ( 6 courses using a combination of CIS, ETP and IFO) was treated by a lobectomy for resection of 2 residual lung metastasis (the nodule in the liver was unsuitable for surgical resection), followed by radiotherapy (12 Gy in both lungs and 12 Gy in the liver), and high-dose conditioning chemotherapy (high-dose chemotherapy

Table 1 - Distribution of Wilms' tumors according to stage and histology.

\begin{tabular}{lcccc}
\hline Tumor Stage & Number & Without Anaplasia & Focal Anaplasia & Diffuse Anaplasia \\
\hline I & 19 & 18 & 1 & 0 \\
II & 14 & 13 & 1 & 0 \\
III & 12 & 9 & 0 & 3 \\
IV & 6 & 5 & 0 & 1 \\
V & 2 & 2 & 0 & 0 \\
Total & 53 & 47 & 2 & 4 \\
\hline
\end{tabular}


- ETP $170 \mathrm{mg} / \mathrm{m}^{2}$, melphalan $140 \mathrm{mg} / \mathrm{m}^{2}$ and CP 600 $\left.\mathrm{mg} / \mathrm{m}^{2}\right)$ with stem cell rescue $\left(2.7 \times 10^{6}\right.$ cells $\left./ \mathrm{kg}\right)(9)$. Autologous CD34 cells were harvested by aphaeresis 45 and 30 days before conditioning therapy after a 5 days course of GM-CSF $(10 \mu \mathrm{g} / \mathrm{kg} / \mathrm{day})$.

The median follow-up was 58 months (range 7 to 274). Survival rates were calculated by the Kaplan-Meier method. Comparisons between groups of patients were made using log rank univariate analysis and Cox regression multivariate analysis. The statistical analysis was performed using Stata ${ }^{\circledR} 6.0$ software. $\mathrm{P}$ values less than 0.05 were considered significant.

\section{RESULTS}

Overall and disease-free survival rates were displayed in Figures- 1 and 2 as well as in Tables-2, 3 and 4. Multivariate analysis shows that influence of tumor stage is more relevant $(\mathrm{p}=0.03$; $\mathrm{HR}=1.56)$ than children age $(\mathrm{p}=0.05 ; \mathrm{HR}=0.39)$ on diseasefree survival rates.

One patient out of 3 with stage I tumor treated with neodjuvant plus adjuvant chemotherapy died of pneumonia 11 months after nephrectomy. The other 2 are alive disease free 240 and 274 months from surgery. The 16 patients with stage I tumor treated with short duration chemotherapy showed a diseasefree survival rate of $100 \%$ in a median time of 101 months (range 14 to 248 months). Only 1 of these 16 patients developed moderate toxicity represented by neutropenia and infection (pneumonia) that was treated successfully by dose reduction and antibiotics.

Ten children showed recurrent WT (Table5 ) in a mean time of $13.4 \pm 10$ months (range 2 to 36). Overall and disease-free survivals of patients with recurrent WT at 3 and 5 years were respectively: $83.3 \%$ and $66.6 \%$, and $42.8 \%$ and $42.8 \%$. Severe drug toxicity in patients with relapse, treated with salvage chemotherapy, occurred in 1 patient that developed cardiac insufficiency. The child treated

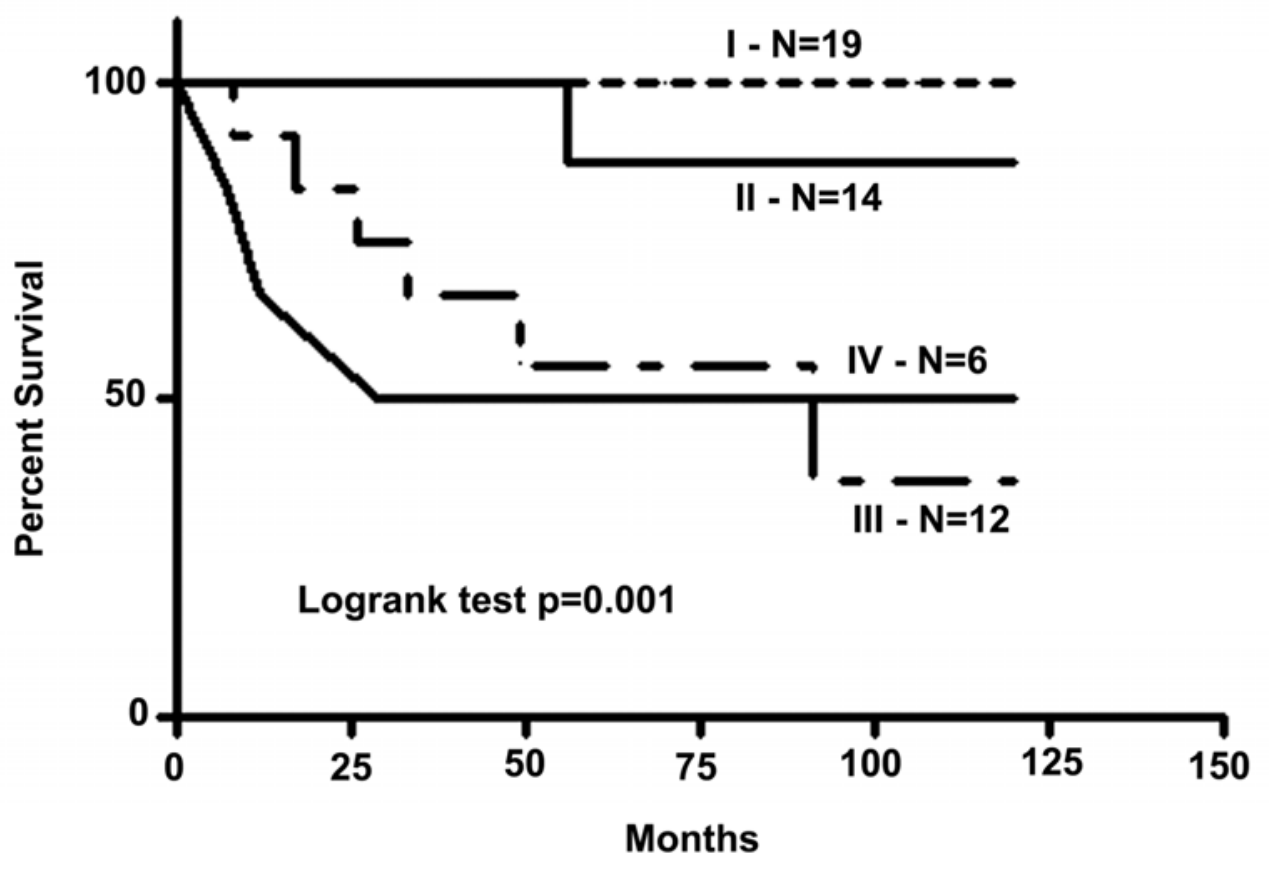

Figure 1 - Disease-free survival rates according to tumor stage. 


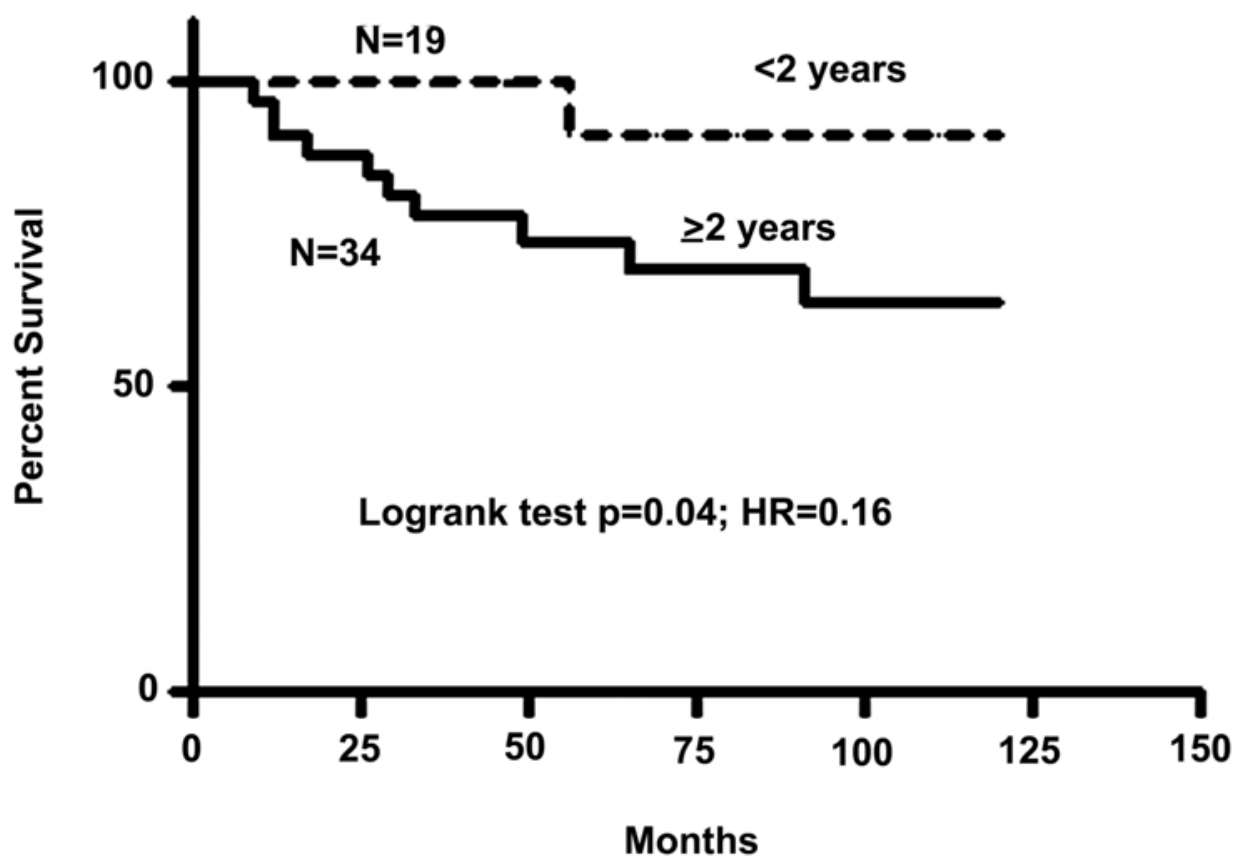

Figure 2 - Influence of age in disease-free survival rates.

Table 2 - Overall and disease-free survival (DFS) rates.

\begin{tabular}{llcl} 
Follow-up & \multicolumn{3}{c}{ Survival Rates (\%) } \\
Sample & 3 years & 5 years & 10 years \\
\hline Overall & $91.5 \pm 4.1$ & $88.2 \pm 5.0$ & $88.2 \pm 5.0$ \\
DFS & $85.7 \pm 5.0$ & $76.7 \pm 6.6$ & $72.8 \pm 7.3$ \\
\hline
\end{tabular}

with high-dose chemotherapy plus stem cell rescue is alive without disease (hepatic metastasis exhibited complete remission) 84 months from relapse, but acute drug toxicity due to this attempt was severe: mucositis, vomiting, diarrhea, seizures, acute pulmonary edema and jaundice. The patient engrafted to an absolute neutrophil count $500 / \mu \mathrm{L}$ and platelet count $>20 / \mu \mathrm{L}$, respectively 18 and 210 days after stem cell transplant.

Only $1 / 16$ adrenal glands excised during radical nephrectomy had WT metastasis (there was no primary tumor contiguous involvement). No major perioperative surgical complication occurred in any children, but 3 cases showed small bowel obstruction

Table 3 - Overall and disease-free survival rates (DFS) according to tumor stage and time of follow-up.

\begin{tabular}{|c|c|c|c|c|}
\hline \multirow[b]{2}{*}{ Tumor Stage } & \multicolumn{2}{|c|}{ Overall Survival Rate (\%) } & \multicolumn{2}{|c|}{ DFS (\%) } \\
\hline & 3 years & 5 years & 3 years & 5 years \\
\hline I & $94.7 \pm 5.1$ & $94.7 \pm 5.1$ & 100 & 100 \\
\hline II & 100 & $87.5 \pm 11.6$ & 100 & $87.5 \pm 11.6$ \\
\hline III & $80.8 \pm 12.2$ & $80.8 \pm 12.2$ & $66.6 \pm 13.6$ & $55.5 \pm 15.2$ \\
\hline IV & $62.5 \pm 21.3$ & $62.5 \pm 21.3$ & $50 \pm 20.4$ & $50 \quad \pm 20.4$ \\
\hline Log rank test & \multicolumn{2}{|c|}{$\mathrm{p}=0.19$} & \multicolumn{2}{|c|}{$\mathrm{p}=0.001$} \\
\hline
\end{tabular}


Table 4 - Disease-free survival rates according to child age and time of follow-up.

\begin{tabular}{lccc}
\hline Follow-up & \multicolumn{3}{c}{ Survival Rates $(\%)$} \\
Age & 3 years & 5 years & 10 years \\
\hline$<2$ years old & 100 & $91.6 \pm 7.9$ & $91.6 \pm 7.9$ \\
$\geq 2$ years old & $78.1 \pm 7.3$ & $73.7 \pm 8.1$ & $63.8 \pm 9.6$ \\
\hline
\end{tabular}

Log rank test $p=0.04 ; H R=0.16$

Table 5 - Place of recurrence versus tumor stage.

\begin{tabular}{lll}
\hline \multirow{2}{*}{ Sites of Recurrence } & \multicolumn{2}{c}{ Tumor Stage } \\
\hline Local/ abdomen & II & III \\
Local/ lungs & 0 & 4 \\
Contralateral kidney / liver & 1 & 1 \\
Lungs & 1 & 0 \\
Liver/ lungs & 0 & 1 \\
Total & 1 & 1 \\
\hline
\end{tabular}

No recurrence occurred in children with stage I tumor.

3, 14 and 20 months after the surgery. Only 1 of these 3 children was treated previously with abdominal radiotherapy. Tumor spill occurred in 5/52 patients: 2 local and 3 diffuse spill. Two children with diffuse spillage exhibited local relapse.

\section{COMMENTS}

Overall and disease-free survival rates after 3 and 5 years of follow-up are within the range reported by others $(7,12-14)$.

Our results show that adjuvant short-duration VCR-AMD chemotherapy is well tolerated and very effective for children of all ages with stage I Wilms' tumor. It is worth to stress that $9 / 16$ children so treated were older than 2 years, no one had diffuse anaplasia and only 1 had focal anaplasia. The SIOP 93-01 trial showed that postoperative chemotherapy with VCRAMD for stage I patients with intermediate-risk and anaplastic WT (submitted to neoadjuvant therapy with the same drugs for 4 weeks and that showed initial good response) can be shortened to 4 weeks from the standard 18 weeks, while maintaining equivalent disease-free survival (4). The UKCCSG showed similar results with adjuvant VCR monotherapy (duration of 10 weeks) for stage I favorable histology tumor in patients $\leq 2$ years (2). The NWTSG-5 trial included a therapy arm in which no adjuvant treatment was given for small stage I WT in children younger than 2 years, but this arm was closed prematurely when the relapse-free survival decreased below $90 \%$ (15). The few existing reports, including our data, suggest that treatment reduction for stage I disease is possible. Meanwhile, the NWTSG still recommend managing patients with stage I disease with a standard adjuvant chemotherapy regimen (VCR plus AMD) for 18 weeks (1).

The outcome of our patients with stage II disease mirrors what was described in the literature while for stages III and IV tumor it seems worse $(3,13,14)$. The difference observed in stages III and IV might be casual or a consequence of disparities in sampling.

Our results show that second line chemotherapy was quite ineffective for treatment of relapsed WT. However, it is relevant to mention that only $1 / 10$ recurrent tumor in this setting fulfilled the favorable prognostic factors such as initial stage I or II, previous treatment with VCR and AMD only, no previous radiotherapy, relapse longer than 6 months after diagnosis and favorable histology (5-7). Although successful retrieval of recurrent tumor is possible, novel approaches are urgently needed. In fact, the treatment for resistant relapsed WT remains a challenge (16). For such patients, in spite of drug toxicity, a salvage attempt with high-dose chemotherapy associated with autologous stem cell transplant seems to be justified as seen in 1 patient of this setting as well as in small series published elsewhere (6,8-10).

The results of transperitoneal radical nephrectomy with regard to tumor spill (10\%), local recurrence $(6 \%)$ and small bowel obstruction $(6 \%)$ are within the range published elsewhere (17-19). The role of systematic adrenalectomy in treatment of WT deserves further evaluation because tumor 
involvement of the gland is not usual as seen in our data.

\section{CONCLUSIONS}

The postoperative chemotherapy in stage I disease can be reduced without compromising the cure rate. The treatment of unfavorable stage III and IV disease or relapsed tumor remains a challenge.

\section{CONFLICT OF INTEREST}

None declared.

\section{REFERENCES}

1. Kalapurakal JA, Dome JS, Perlman EJ, Malogolowkin M, Haase GM, Grundy P, et al.: Management of Wilms' tumour: current practice and future goals. Lancet Oncol. 2004; 5: 37-46.

2. Pritchard-Jones K, Kelsey A, Vujanic G, Imeson J, Hutton C, Mitchell C, et al.: Older age is an adverse prognostic factor in stage I, favorable histology Wilms' tumor treated with vincristine monochemotherapy: a study by the United Kingdom Children's Cancer Study Group, Wilm's Tumor Working Group. J Clin Oncol. 2003; 21:3269-75.

3. D'Angio GJ, Breslow N, Beckwith JB, Evans A, Baum H, deLorimier A, et al.: Treatment of Wilms' tumor. Results of the Third National Wilms' Tumor Study. Cancer. 1989; 64: 349-60.

4. de Kraker J, Graf N, van Tinteren H, Pein F, Sandstedt B, Godzinski J, et al.: Reduction of postoperative chemotherapy in children with stage I intermediate-risk and anaplastic Wilms' tumour (SIOP 93-01 trial): a randomised controlled trial. Lancet. 2004; 364: 1229-35.

5. Grundy P, Breslow N, Green DM, Sharples K, Evans A, D'Angio GJ: Prognostic factors for children with recurrent Wilms' tumor: results from the Second and Third National Wilms' Tumor Study. J Clin Oncol. 1989; 7:638-47.

6. Dome JS, Liu T, Krasin M, Lott L, Shearer P, Daw NC, et al.: Improved survival for patients with recurrent Wilms tumor: the experience at St. Jude Children's Research Hospital. J Pediatr Hematol Oncol. 2002; 24 : 192-8.
7. Weirich A, Ludwig R, Graf N, Abel U, Leuschner I, Vujanic GM, et al.: Survival in nephroblastoma treated according to the trial and study SIOP-9/GPOH with respect to relapse and morbidity. Ann Oncol. 2004; 15: 808-20.

8. Garaventa A, Hartmann O, Bernard JL, Zucker JM, Pardo N, Castel V, et al.: Autologous bone marrow transplantation for pediatric Wilms' tumor: the experience of the European Bone Marrow Transplantation Solid Tumor Registry. Med Pediatr Oncol. 1994; 22: 11-4.

9. Pein F, Michon J, Valteau-Couanet D, Quintana E, Frappaz D, Vannier JP, et al.: High-dose melphalan, etoposide, and carboplatin followed by autologous stem-cell rescue in pediatric high-risk recurrent Wilms' tumor: a French Society of Pediatric Oncology study. J Clin Oncol. 1998; 16: 3295-301.

10. Campbell AD, Cohn SL, Reynolds M, Seshadri R, Morgan E, Geissler G, et al.: Treatment of relapsed Wilms' tumor with high-dose therapy and autologous hematopoietic stem-cell rescue: the experience at Children's Memorial Hospital. J Clin Oncol. 2004; 22: 2885-90.

11. Bonadio JF, Storer B, Norkool P, Farewell VT, Beckwith JB, D'Angio GJ: Anaplastic Wilms' tumor: clinical and pathologic studies. J Clin Oncol. 1985; 3: 513-20.

12. de Camargo B, Franco EL: A randomized clinical trial of single-dose versus fractionated-dose dactinomycin in the treatment of Wilms' tumor. Results after extended follow-up. Brazilian Wilms' Tumor Study Group. Cancer. 1994; 73: 3081-6.

13. Green DM, Breslow NE, Beckwith JB, Finklestein JZ, Grundy P, Thomas PR, et al.: Effect of duration of treatment on treatment outcome and cost of treatment for Wilms' tumor: a report from the National Wilms' Tumor Study Group. J Clin Oncol. 1998; 16: 3744-51.

14. Pianezza ML, Rubin S, Bass J, Chou S, Pike JG, Leonard MP: Wilms' tumor at the Children's Hospital of Eastern Ontario: 1990-2001. Can J Urol. 2004; 11: 2151-6.

15. Green DM, Breslow NE, Beckwith JB, Ritchey ML, Shamberger RC, Haase GM, et al.: Treatment with nephrectomy only for small, stage I/favorable histology Wilms' tumor: a report from the National Wilms' Tumor Study Group. J Clin Oncol. 2001; 19: 3719-24.

16. Grundy P, Perlman E, Rosen NS, Warwick AB, Bender JG, Ehrlich P, et al.: Current issues in Wilms tumor management. Curr Probl Cancer. 2005; 29: 221-60.

17. Ehrlich PF, Ritchey ML, Hamilton TE, Haase GM, Ou S, Breslow N, et al.: Quality assessment for Wilms' 
tumor: a report from the National Wilms' Tumor Study5. J Pediatr Surg. 2005; 40: 208-12.

18. Seseke F, Zoller G, Ringert RH: Wilms' tumor-singlecenter experience with renal surgery. Scand J Urol Nephrol. 2004; 38: 373-7.
19. Ritchey ML, Shamberger RC, Haase G, Horwitz J, Bergemann T, Breslow NE: Surgical complications after primary nephrectomy for Wilms' tumor: report from the National Wilms' Tumor Study Group. J Am Coll Surg. 2001; 192: 63-8.
Accepted after revision:

October 15, 2006

\author{
Correspondence address: \\ Dr. Silvio Tucci Jr \\ Hospital das Clínicas de Ribeirão Preto \\ University of São Paulo \\ Av. Bandeirantes, 3900 \\ Ribeirão Preto, SP, 14048-900, Brazil \\ E-mail: stucci@convex.com.br
}

\section{EDITORIAL COMMENT}

The management of Wilms' Tumor (WT) over the last two decades has seen vast improvements in overall survival due to better neoadjuvant and adjuvant therapeutic protocols developed by national and international collaborations (1). With the survival improvements, came the need to reduce treatment intensity so that the health burden on patients, parents and the health care system was reduced without compromising outcome. The Brazilian collaborative group (Grupo Cooperativo Brasileiro para o Tratamento do Tumor de Wilms [GCBTTW]) (2) contributed to this international consensus by showing that a single day administration of actinomycin-D rather than a 5-day course (provided other treatment regimens were constant) was equally effective.

Tucci et al., in this article, provide a retrospective summary of 53 children treated at a single centre over a 14-year period. Although the retrospective nature of the study is recognized, the authors should be congratulated on providing further evidence that stage-I WT need not aggressive chemotherapeutic regimens. Almost all of these patients did not have unfavorable histology and thus the reduction in chemotherapy seems to be the correct regimen for them. Such results are supported by the European and British groups, both of whom have shown reduction of chemotherapy does not necessarily lead to poorer outcome. The UK group showed that overall survival was in the high nineties when vincristine was given only over ten weeks. However, this applied to only those 4 years of age or younger (3). The SIOP have reduced the pre-operative chemotherapy to 4 weeks and shown it is as good as 8 weeks with no change of stage distribution and tumor shrinkage. Tucci et al., in the present paper, mention the recent NWTSG-5 study showing that of 75 patients younger than 2 years with a stage-I, favorable histology WT less than 550g in weight treated without adjuvant chemotherapy 8 patients developed recurrence to the lung or operative bed and 3 developed metachronous contralateral WT. This resulted in a 2-year disease-free survival estimate of $86.5 \%$. Based on predefined stopping rules, this arm of the study was closed early. However, subsequent 
review of these patients revealed several factors that were not considered in these predefined stopping rules, the most important being that the overall survival rate of these patients was much higher than estimated. This suggested that, even if these children relapse, the ability to successfully control the relapse was far greater than predicted. Based on this, the newly formed US Children's Oncology Group will again evaluate this question in this group (4).

The outcome for relapsed WT is worrying with intensive treatment having variable effect. Certainly, this cohort seems to have worse outcome compared to that reported elsewhere, but with the patients recruited over a period of 14 years, many of whom were treated before more recent studies, there is likely to be heterogeneity in management and possibly even histological evaluation. The authors may have given consideration to a review of all histology by expert histopathologists and application of recent staging criteria so that such effects could be evaluated. The future for these patients may be in high-dose chemotherapy with autologous stem cell transplant, but as exemplified by the one patient they treated the toxicity of treatment can be distressing for all concerned. Recent molecular studies have started to characterize genetic changes that may stratify relapses into high and low risk (5). Such advances could

\section{EDITORIAL COMMENT}

I read with great interest the article titled "Results of Novel Strategies for the Treatment of Wilms' Tumor" and I would like to congratulate Silvio identify subgroups that may have higher risk of failure after attempted salvage with intensive chemotherapeutic regimens. In such patients, the risk: benefit ratio may be more appealing when considering autologous stem-cell transplant and chemotherapy.

\section{REFERENCES}

1. Ahmed HU, Arya M, Tsiouris A, Sellaturay SV, Shergill IS, Duffy PG, et al.: Update on the management of Wilms' tumour. Eur J Surg Oncol. 2007; Feb 19, [Epub ahead of print].

2. de Camargo B, Franco EL: A randomized clinical trial of single-dose versus fractionated-dose dactinomycin in the treatment of Wilms' tumor. Results after extended follow-up. Brazilian Wilms' Tumor Study Group. Cancer. 1994; 73: 3081-6.

3. Mitchell C, Morris-Jones P, Kelsey A, Vujanic GM, Marsden B, Shannon R, et al.: The treatment of Wilms' tumour: results of the United Kingdom children's cancer study group (UKCCSG) second Wilms' tumour study. Br J Cancer. 2000; 83 :602-8.

4. Perlman EJ: Pediatric renal tumors: practical updates for the pathologist. Pediatr Dev Pathol. 2005;8: 320-38.

5. Natrajan R, Little SE, Sodha N, Reis-Filho JS, Mackay A, Fenwick K, et al.: Analysis by array CGH of genomic changes associated with the progression or relapse of Wilms' tumour. J Pathol. 2007; 211: 52-9.

\section{Dr. Hashim U Ahmed Dr. Manit Arya Dr. Imran Mushtaq}

Great Ormond Street Hospital for Children The Institute of Urology \& Nephrology Division of Surgical and Interventional Sciences University College London, UK E-mail: hashim.ahmed@ucl.ac.uk

Tucci Jr et al. The article represents a critical, in-depth contribution to the issue of contemporary Wilms' tumor treatment.

Wilms' tumor, represents approximately $6 \%$ of all childhood cancers and is the most common 
primary malignant renal tumor of childhood. Current management emphasizes in reducing the morbidity of treatment for low-risk patients and reserving more intensive treatment for selected high-risk patients for whom survival remains poor.

There is a well known debate going on according to the treatment protocol one must use. The International Society of Pediatric Oncology (SIOP) Protocols have always recommended preoperative chemotherapy because it is able to reduce tumor size, induce a pseudocapsule and decrease the incidence of tumor rupture (1). Indeed, the authors describe an above average tumor rupture in their study. The National Wilms' Tumor Study Group (NWTSG), on the other hand, recommends preoperative chemotherapy in some cases only; bilateral tumors, inoperable tumors at surgical exploration and inferior vena cava extension above the hepatic veins. This allows precise staging of patients with modulation of treatment for each individual, thereby decreasing the intensity of treatment toxicity. The authors have chosen the NWTSG protocol, while in Europe the SIOP protocol is more popular and therefore mostly used.

I would also like to congratulate the author's surgical approach. I believe that transperitoneal radical nephrectomy with regional lymph node sampling is the optimal surgical approach for Wilms' tumor patients, since it allows complete inspection of abdominal cavity, lymph node sampling and tumor resection with lower percentage rates of neoplastic cell spillage (2).

Reduced postoperative chemotherapy seems to be a good solution in stage I patients since their cure rate is not compromised. High dose chemotherapy with autologous stem-cell rescue in children with relapsed Wilms' tumor exhibits variable disease free survival rates and needs to be further studied since it is not clear if it offers any advantages over conventional second line therapies. Treatment intensification for children with high-risk tumors, although accompanied with sever complications, seems to be at the time the only solution for such patients.

\section{REFERENCES}

1. Graf N, Tournade MF, de Kraker J: The role of preoperative chemotherapy in the management of Wilms tumor. The SIOP studies. International Society of Pediatric Oncology. Urol Clin North Am. 2000; 27: 443-54.

2. Zugor V, Krot D, Schott GE: Risk factors for intra- and postoperative complications in Wilms' tumor surgery. Urologe A. 2007; 46: 274-7.

Dr. Vahudin Zugor

Department of Urology

Friedrich-Alexander-Universitat

Erlangen-Nurnberg

Erlangen, Germany

E-mail:vahudin.zugor@uro.imed.uni-erlangen.de 